\title{
Random Attractor Family for a Class of Stochastic Higher-Order Kirchhoff Equations
}

\author{
Guoguang Lin $^{1}$, Liping Guan ${ }^{2}$ \\ ${ }^{1}$ Department of Mathematics, Yunnan University, Kunming, Yunnan 650091, People's Republic of China \\ ${ }^{2}$ Department of Mathematics, Yunnan University, Kunming, Yunnan 650091, People's Republic of China \\ Correspondence: Department of Mathematics, Yunnan University, Kunming, Yunnan 650091, People's Republic of China. \\ E-mail: gglin@ynu.edu.cn
}

Received: March 27, 2019 Accepted: April 22, 2019 Online Published: May 8, 2019

doi:10.5539/jmr.v11n3p23

URL: https://doi.org/10.5539/jmr.v11n3p23

\begin{abstract}
A class of stochastic dynamical systems with strong damped stochastic higher order Kirchhoff equation solutions with white noise is studied. Firstly, the equation is transformed into a stochastic equation with random variables as parameters and without noise by using Ornstein-Uhlenbeck process. Secondly, the bounded stochastic absorption set is obtained by estimating the solution of the equation. Finally, the stochastic dynamical system is obtained by using the isomorphic mapping method and the compact embedding theorem. It is progressively compact, thus proving the existence of random attractors.
\end{abstract}

Keywords: stochastic Kirchhoff equation, random attractor family, Wiener process, Ornstein-Uhlenbeck process

2010 MSC: 35K10, 35K25, 35K35

\section{Introduction}

In this paper, the stochastic higher-order Kirchhoff equation with strong damping and additive noise is studied.

$$
\begin{gathered}
u_{t t}+M\left(\left\|D^{m} u\right\|^{2}\right)(-\Delta)^{m} u+\beta(-\Delta)^{m} u_{t}+\Delta g(u)=q(x) \dot{W}, \\
u(x, t)=0, \quad \frac{\partial^{i} u}{\partial v^{i}}=0, \quad i=1,2, \cdots, m-1, \quad x \in D, \quad t \in[0,+\infty), \\
u(x, 0)=u_{0}(x), \quad u_{t}(x, 0)=u_{1}(x) .
\end{gathered}
$$

Where $m>1, \Delta g(u)$ is a second-order non-linear source term, $M$ is a real-valued function, $\beta>0 . u=u(x, t)$ is a real-valued function on $D \times[0,+\infty), D$ is a bounded open set with smooth boundary on $R^{n}(n \in N)$. $q d W$ describes an additive white noise. $W(t)$ is a one-dimensional bilateral Wiener process on probability space $(\Omega, F, P)$, $\Omega=\{\omega \in C(R, R): \omega(0)=0\}, F$ is a Borel algebra generated by compact expansion on $\Omega$ and $P$ is a probability measure.

Chuangliang Qin, Jinji Du (2016) have studied the stochastic low-order Kirchhoff equation with strong damping and additive noise.

$$
\begin{gathered}
d u_{t}+\left(-\alpha \Delta u_{t}+\beta u_{t}-\left(1+\left(\int_{\Omega}|D u|^{2} d x\right)^{p}\right) \Delta u+g(u)\right) d t=f(x) d t+q(x) d W(t), x \in D, t \in[0,+\infty) \\
\left.u(x, 0)\right|_{x \in \supset D}=0, u(x, 0)=u_{0}(x), u_{t}(x, 0)=u_{1}(x) .
\end{gathered}
$$

By using Ornstein-Uhlenbeck process and isomorphic mapping method, the existence and uniqueness of solutions and the existence of random attractors for stochastic Kirchhoff equation with strong damping are obtained.

Guigui Xu, Libo Wang and Guoguang Lin (2017) discussed the nonautonomous stochastic wave equation with dispersion term and dissipation term.

$$
\begin{gathered}
u_{t t}-\Delta u-\alpha \Delta u_{t}-\beta u_{t t}+h(u) u_{t}+\lambda u+f(x, u)=g(x, t) u+\varepsilon u \cdot \frac{d W}{d t} \\
u(x, \tau)=u_{0}(x), u_{t}(x, \tau)=u_{1}(x) .
\end{gathered}
$$


The existence of random attractors for Nonautonomous stochastic wave equations with product white noise is obtained by using the uniform estimation of solutions and the technique of decomposing solutions in a region.

Meng Wang (2017)analyzed autonomous and non-autonomous stochastic wave equation with strong damping

$$
\begin{aligned}
& u_{t t}-\alpha \Delta u_{t}+u_{t}+f(u)-\Delta u=g(x) \frac{d W}{d t}, t \in[0,+\infty), \\
& \left.u(x, t)\right|_{x \in \partial U}=0, t \geq 0, \quad u(x, 0)=u_{0}(x), u_{t}(x, 0)=u_{1}(x), x \in U .
\end{aligned}
$$

and

$$
\begin{aligned}
& u_{t t}-\alpha \Delta u_{t}+u_{t}+f(u)-\Delta u=g(x)+c u \cdot \frac{d W}{d t}, t \in[0,+\infty), \\
& \left.u(x, t)\right|_{x \in \partial U}=0, t \geq 0, \quad u(x, 0)=u_{0}(x), u_{t}(x, \tau)=u_{1}(x), x \in U .
\end{aligned}
$$

The existence of random attractors and the upper bound of fractal dimension are discussed. First, they deal with random terms by using Ornstein-Uhlenbeck process and weak solutions of the established equation. Then, the solutions of the equation are estimated and bounded random absorption sets are obtained. Finally, the system is asymptotically compact by using the tight embedding theorem, which proves the existence of random attractors.

Donghong Cai and Xiaoming Fan (2014) considered the dissipative KdV equation with multiplicative noise

$$
\begin{aligned}
d u= & \left(a u_{x x x}+u_{x x}+\beta u u_{x x}+r u\right) d t=f(x) d t+b u d W(t), x \in D, t>0, \\
& u(x, 0)=u_{t_{0}} x, x \in D, \\
& u(0, t)=u(L, t)=0 .
\end{aligned}
$$

The equation is transformed into a stochastic $\mathrm{KdV}$-type equation without white noise by appropriate transformation. In the new equation, the sample can be regarded as a common parameter. By using the methods and techniques of determining the KdV-type equation and the slowly increasing property of Wiener process, the absorptivity and asymptotic compactness of the dynamic system determined by the new equation are obtained, thus proving the existence of the stochastic attractor of the new equation. The stochastic attractor of the dynamic system determined by the dissipative KdV equation of multiplicative white noise also exists.

As a deterministic system, stochastic dynamical systems have been extensively studied. Specific cases can be referred to in references(Qin, C. L., Du, J. J., \&Lin, G. G.2017; Lin, G. G., Chen, L., \&Wang, W.2017; Guo, B. L.2000; Guo, B. L., \& Pu, X. K.2009; Lin, G. G.2011; Massatt, P.1988; Guo, B. L., \&Wang, B. X.1995; Zhao, C. D., \&Zhou, S. F.2009;Yin, J. Y., Li, Y. R., \&Zhao, H. J.2013; Wang, R., \&Li, Y. R.2012; Hao, H. J., \&Zhou, S. F.2010; Cheng, Y. Y., \&Li, Y. R.2012 ).

On the basis of the correlation of random attractors for some low-order Kirchhoff equation with additive noise stochastic studied by previous scholars, the existence and uniqueness of solutions for high-order Kirchhoff equation with strong damping stochastic with additive noise are discussed, and the existence of attractors for stochastic Kirchhoff equation with strong damping is proved. This paper is organized as follows. Section 2, introduces the assumptionsbasic and basic knowledge needed in this paper. Section 3, discuss the existence and uniqueness of solutions of stochastic high-order Kirchhoff equation with strong damping and additive noise, and prove the existence of random attractors.

\section{Preliminaries}

In this section, we introduce some basic assumptions and knowledge of stochastic dynamical systems required in this paper.

For the sake of narrative convenience, we introduce the following symbols:

$\nabla=D, H=L^{2}(\Omega), H_{0}^{m}(\Omega)=H^{m}(\Omega) \cap H_{0}^{1}(\Omega), H_{0}^{m+k}(\Omega)=H^{m+k}(\Omega) \cap H_{0}^{1}(\Omega) E_{k}=H_{0}^{m+k}(\Omega) \times H_{0}^{k}(\Omega),(k=0,1,2, \cdots m)$.

The following norms and inner products are defined:

$$
\begin{gathered}
(u, v)=\int_{\Omega} u v d x, \quad\|u\|=(u, u)^{\frac{1}{2}}, \quad \forall u, v \in H_{0}^{k}(\Omega) . \\
\left(y_{1}, y_{2}\right)_{E_{k}}=\left(D^{m+k} u_{1}, D^{m+k} u_{2}\right)+\left(D^{k} v_{1}, D^{k} v_{2}\right) . \quad \forall y_{i}=\left(u_{i}, v_{i}\right) \in E_{k} \quad i=1,2 .(k=1,2, \cdots m) .
\end{gathered}
$$

(H1) Assume that the Kirchhoff type stress term satisfies: 


$$
\left.\varepsilon+1<\mu_{0} \leq M(s) \leq \mu_{1} .{ }_{0<\varepsilon<\min \left\{\frac{\sqrt{4+2 \beta \lambda_{1}^{m}}-2}{2}\right.}, \frac{\mu_{0}+\sqrt{\mu_{0}^{2}-\frac{2 C_{0}^{2}}{\lambda_{1}^{2 m-2}}}}{\beta}, \frac{1}{\beta-1}\right\} .
$$

Where $\mu_{0}, \mu_{1}$ are constants, $\lambda_{1}$ is the first eigenvalue of $-\Delta$ with homogeneous Dirichlet boundary conditions on $\Omega$.

(H2) Assume that the non-linear source term satisfies:

$$
g(s) \in C^{3}(R) \text { and }\left\|g^{\prime}(s)\right\|_{\infty}=C_{0} \leq \frac{\sqrt{2}}{2} \lambda_{1}^{m-1} .\left(\lambda_{1}>0\right) .
$$

Let $(\Omega, F, P)$ be a probabilistic space and define a family of measures-preserving and ergodic transformations $\left\{\theta_{t}, t \in R\right\}$ :

$$
\theta_{t} w(\cdot)=w(\cdot+t)-w(t),
$$

$\left(\Omega, F, P,\left(\theta_{t}\right)_{t \in R}\right)$ is an ergodic metric dynamical system.

Let $\left(X,\|\|_{X}\right)$ be a complete separable metric space and $B(X)$ be a Borel $\sigma$-algebra over $X$.

Definition $1^{\text {[Chuangliang Qin, Jinji Du, \&Guoguang Lin(2017)] } L e t ~}\left(\Omega, F, P,\left(\theta_{t}\right)_{t \in R}\right)$ be a metric dynamical system, if $\left(B\left(R^{+}\right) \times F \times B(X), B(X)\right)$ measurable mapping

$$
S: R^{+} \times \Omega \times X \rightarrow X, \quad(t, w, x) \mapsto S(t, w, x) .
$$

satisfaction

(1) for all $s, t \geq 0$ and $w \in \Omega$, mapping $S(t, w):=S(t, w, \cdot)$ satisfies

(2) for each $w \in \Omega$,mapping $(t, w) \mapsto S(t, w, x)$ is continuous.

$$
S(0, w)=i d, \quad S(t+s, w)=S\left(t, \theta_{s} w\right) \circ S(s, w),
$$

$S$ is a continuous stochastic dynamical system on $\left(\Omega, F, P,\left(\theta_{t}\right)_{t \in R}\right)$.

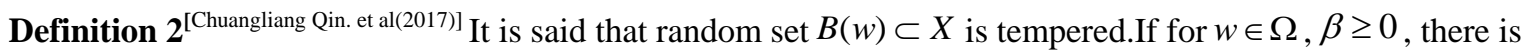

$$
\lim _{|s| \rightarrow \infty} \inf e^{-\beta s} d\left(B\left(\theta_{-s} w\right)\right)=0
$$

Where $^{d(B)}=\sup _{x \in B}\|x\|_{X}$, for $\forall x \in X$.

Definition $3^{\text {[Chuangliang Qin. et al(2017)] }}$ Record $D(w)$ as the set of all random sets on $X$, a random set $B(w)$ is called an absorption set on $D(w)$.If for any $B(w) \in D(w)$ and $P_{\text {a.e. }} \in \Omega$, there exists $T_{B(w)}>0$ such that

$$
S\left(t, \theta_{-t} w\right)\left(B\left(\theta_{-t} w\right)\right) \subset B_{0}(w) .
$$

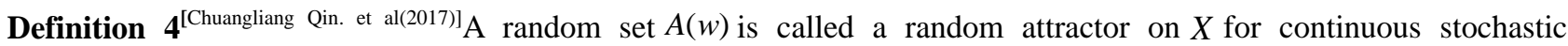
dynamical system $S(t)$.If random set $A(w)$ satisfies

(1) $A(w)$ is a random compact set;

(2) $A(w)$ is invariant set $D(w)$, that is, to arbitrary $t>0, S(t, w) A(w)=A\left(\theta_{t} w\right)$;

(3) $A(w)$ attracts every sets in $D(w)$, that is, for any $B(w) \in D(w)$ and $P_{\text {a.e. }} \in \Omega$, we have the limit formula

$$
\lim _{t \rightarrow \infty} d\left(S\left(t, \theta_{-t} w\right) B\left(\theta_{-t} w\right), A(w)\right)=0 .
$$

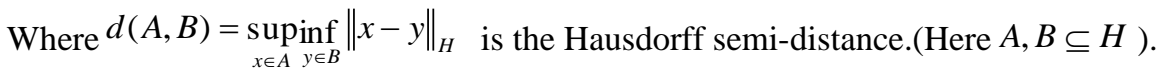

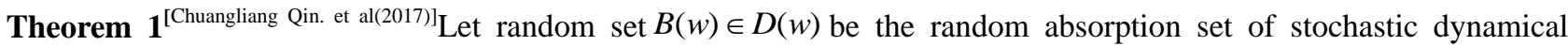
$\operatorname{system}(S(t, w))_{t \geq 0}$, and random set $B(w)$ satisfies

(1) random set $B(w)$ is a closed set on Hilbert space;

(2) for $P_{\text {a.e.w }} \in \Omega$, random set $B(w)$ satisfies the following asymptotic compactness conditions 
for arbitrary sequence $x_{n} \in S\left(t_{n}, \theta_{-t_{n}} w\right) B_{0}\left(\theta_{-t_{n}} w\right), t_{n} \rightarrow+\infty$, there is a convergent subsequence in space $X$, Then the stochastic dynamical system $(S(t, w))_{t \geq 0}$ has a unique global attractor.

$$
A(w)=\bigcap_{\tau \geq t(w) t \geq \tau} \overline{U S\left(t, \theta_{-t} w\right) B_{0}\left(\theta_{-t} w\right)} .
$$

Ornstein-Uhlenbeck process ${ }^{\text {[Chuangliang Qin. et al(2017)] }}$

In this section, space is introduced. Ornstein-Uhlenbeck process on $H_{0}^{m+k}(\Omega)$, where O-U process is given by Wiener process on measurement system $\left(\Omega, F, P,\left(\theta_{t}\right)_{t \in R}\right)$.

Set $z\left(\theta_{t} w\right)=-\alpha \int_{-\infty}^{0} e^{\alpha \tau} \theta_{t} w(\tau) d \tau$, where $t \in R$. It can be seen that for arbitrary $t \geq 0$, the stochastic process $z\left(\theta_{t} w\right)$ satisfies the Itô equation.

$$
d z+\alpha z d t=d W(t)
$$

According to the properties of $\mathrm{O}-\mathrm{U}$ process, there exists a probability measure $P, \theta_{t}$-invariant set $\Omega_{0} \subset \Omega$; and the above stochastic process $z\left(\theta_{t} w\right)=-\alpha \int_{-\infty}^{0} e^{\alpha \tau} \theta_{t} w(\tau) d \tau$, satisfies the following properties

(1) for any given $w \subset \Omega_{0}$, the mapping $s \rightarrow z\left(\theta_{s} w\right)$ is a continuous mapping;

(2) random variable $\|z(w)\|$ is slowly increasing;

(3) there is a slowly increasing $r(w)>0$, so that $\left\|z\left(\theta_{t} w\right)\right\|+\left\|z\left(\theta_{t} w\right)\right\|^{2} \leq r\left(\theta_{t} w\right) \leq r(w) e^{\frac{\alpha}{2}|t|}$;

(4) $\lim _{t \rightarrow \infty} \frac{1}{t} \int_{0}^{t}\left|z\left(\theta_{t} w\right)\right|^{2} d \tau=\frac{1}{2 \alpha}$;

(5) $\lim _{t \rightarrow \infty} \frac{1}{t} \int_{0}^{t}\left|z\left(\theta_{t} w\right)\right| d \tau=\frac{1}{\sqrt{\pi \alpha}}$.

\section{Existence of Random Attractor Family}

In this section, we discuss the existence and uniqueness of solutions of stochastic high-order Kirchhoff equation with strong damping and additive noise, and prove the existence of random attractors.

For convenience, equation (1.1)-(1.3) can be transformed into

$$
\left\{\begin{array}{l}
d u=u_{t} d t \\
d u_{t}+\left[M\left(\left\|A^{\frac{m}{2}} u\right\|^{2}\right) A^{m} u+\beta A^{m} u_{t}+\Delta g(u)\right] d t=q(x) d W(t), t \in[0,+\infty) \\
u(x, 0)=u_{0}(x), u_{t}(x, 0)=u_{1}(x), x \in \Omega .
\end{array}\right.
$$

where $A=-\Delta$.

Let $\varphi=(u, v)^{T}, v=u_{t}+\varepsilon u$, then the problem (3.1) can be simplified to

$$
\left\{\begin{array}{l}
d \varphi+L \varphi d t=F\left(\theta_{t} \omega, \varphi\right) \\
\varphi_{0}(\omega)=\left(u_{0}, u_{1}+\varepsilon u_{0}\right)^{T}
\end{array}\right.
$$

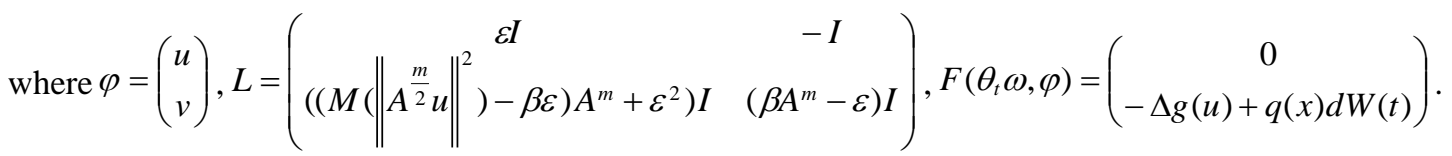

If $z=v-q(x) \delta\left(\theta_{t} \omega\right)$, then equestion (3.1) can be written as

$$
\left\{\begin{array}{l}
\psi_{t}+L \psi=\bar{F}\left(\theta_{t} \omega, \psi\right) \\
\psi_{0}(\omega)=\left(u_{0}, u_{1}+\varepsilon u_{0}-q(x) \delta\left(\theta_{t} \omega\right)\right)^{T}
\end{array}\right.
$$




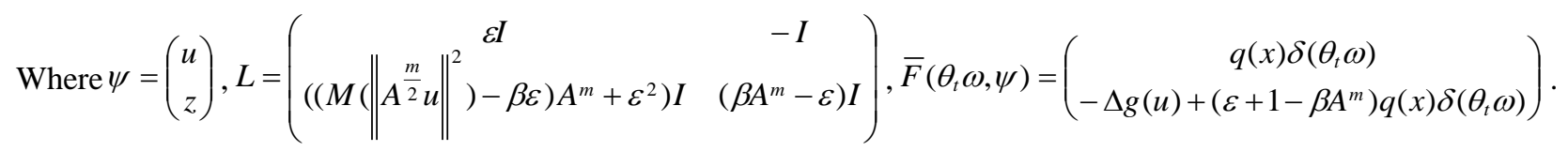

Lemma 1 Assuming that the Kirchhoff stress term and the non-linear term satisfy the conditions (H1), (H2), respectively. $f \in H,\left(u_{0}, v_{0}\right) \in E_{0}$, Then the initial boundary value problem (1.1) - (1.3) has smooth solutions $(u, v) \in E_{0}$ and satisfies the following inequalities

$$
\|(u, v)\|_{E_{0}}^{2}=\left\|D^{m} u\right\|^{2}+\|v\|^{2} \leq\left(\left\|v_{0}\right\|^{2}+\mu\left\|D^{m} u_{0}\right\|^{2}+\varepsilon^{2}\left\|u_{0}\right\|^{2}\right) e^{-\alpha_{1} t}+\frac{C_{1}}{\alpha_{1}}\left(1-e^{-\alpha_{1} t}\right) .
$$

Where $v=u_{t}+\varepsilon u, \alpha_{1}=\min \left\{a_{1}, \frac{a_{2}}{\mu}, 2 \varepsilon\right\}$, there exiet $C\left(R_{1}\right)$ and $t=t_{1}(\Omega)>0$, such that

$$
\|(u, v)\|_{E_{0}}^{2}=\left\|D^{m} u\right\|^{2}+\|v\|^{2} \leq C\left(R_{1}\right) . \quad\left(t>t_{1}\right) .
$$

Proof Let $v=u_{t}+\varepsilon u, v$ and the two sides of equation (1.1) are used as inner products, we can get

$$
\left(u_{t t}+M\left(\left\|D^{m} u\right\|^{2}\right)(-\Delta)^{m} u+\beta(-\Delta)^{m} u_{t}+\Delta g(u), v\right)=(q(x) \dot{W}, v) .
$$

From Holder's inequality, Young's inequality and Poincare's inequality, we can get

$$
\left(u_{t t}, v\right)=\frac{1}{2} \frac{d}{d t}\|v\|^{2}-\varepsilon\|v\|^{2}+\frac{\varepsilon^{2}}{2} \frac{d}{d t}\|u\|^{2}+\varepsilon^{3}\|u\|^{2} .
$$

From hypothesis (H1), we can get

$$
\left(M\left(\left\|D^{m} u\right\|^{2}\right)(-\Delta)^{m} u, v\right)=\left(M\left(\left\|D^{m} u\right\|^{2}\right) \frac{1}{2} \frac{d}{d t}\left\|D^{m} u\right\|^{2}+\varepsilon M\left(\left\|D^{m} u\right\|^{2}\right)\left\|D^{m} u\right\|^{2} .\right.
$$

The following two aspects are discussed

$$
\begin{aligned}
& \text { (1)When } \frac{1}{2} \frac{d}{d t}\left\|D^{m} u\right\|^{2} \geq 0 \text {, it can be obtained by hypothesis (H1). } \\
& \qquad\left(M\left(\left\|D^{m} u\right\|^{2}\right) \frac{1}{2} \frac{d}{d t}\left\|D^{m} u\right\|^{2}+\varepsilon M\left(\left\|D^{m} u\right\|^{2}\right)\left\|D^{m} u\right\|^{2} \geq \frac{\mu_{0}}{2} \frac{d}{d t}\left\|D^{m} u\right\|^{2}+\varepsilon \mu_{0}\left\|D^{m} u\right\|^{2} .\right.
\end{aligned}
$$

Let $\mu=\mu_{0}$, there are

$$
\left(M\left(\left\|D^{m} u\right\|^{2}\right)(-\Delta)^{m} u, v\right) \geq \frac{\mu}{2} \frac{d}{d t}\left\|D^{m} u\right\|^{2}+\varepsilon \mu_{0}\left\|D^{m} u\right\|^{2} .
$$

(2) When $\frac{1}{2} \frac{d}{d t}\left\|D^{m} u\right\|^{2}<0$, it can be obtained by hypothesis (H1).

$$
\left(M\left(\left\|D^{m} u\right\|^{2}\right) \frac{1}{2} \frac{d}{d t}\left\|D^{m} u\right\|^{2}+\varepsilon M\left(\left\|D^{m} u\right\|^{2}\right)\left\|D^{m} u\right\|^{2} \geq \frac{\mu_{1}}{2} \frac{d}{d t}\left\|D^{m} u\right\|^{2}+\varepsilon \mu_{0}\left\|D^{m} u\right\|^{2} .\right.
$$

Let $\mu=\mu_{1}$, there are

$$
\left(M\left(\left\|D^{m} u\right\|^{2}\right)(-\Delta)^{m} u, v\right) \geq \frac{\mu}{2} \frac{d}{d t}\left\|D^{m} u\right\|^{2}+\varepsilon \mu_{0}\left\|D^{m} u\right\|^{2} .
$$

Comprehensive (3.9) - (3.12), we have

$$
\begin{aligned}
& \left(M\left(\left\|D^{m} u\right\|^{2}\right)(-\Delta)^{m} u, v\right) \geq \frac{\mu}{2} \frac{d}{d t}\left\|D^{m} u\right\|^{2}+\varepsilon \mu_{0}\left\|D^{m} u\right\|^{2} . \\
& \left(\beta(-\Delta)^{m} u_{t}, v\right) \geq \frac{\beta \lambda_{1}^{m-1}}{4}\|D v\|^{2}+\frac{\beta \lambda_{1}^{m}}{4}\|v\|^{2}-\frac{\beta \varepsilon^{2}}{2}\left\|D^{m} u\right\|^{2} .
\end{aligned}
$$

From hypothesis (H2), we can get 


$$
\begin{gathered}
|(\Delta g(u), v)| \geq-\left\|\mathrm{g}^{\prime}(u)\right\|_{\infty} \cdot\|D u\| \cdot\|D v\| \geq-\frac{\beta \lambda_{1}^{m-1}}{4}\|D v\|^{2}-\frac{C_{0}^{2}}{\beta \lambda_{1}^{2(m-1)}}\left\|D^{m} u\right\|^{2} . \\
(q(x) \dot{W}, v) \leq\|q(x) \dot{W}\| \cdot\|v\| \leq \frac{1}{2 \varepsilon^{2}}\|q(x) \dot{W}\|^{2}+\frac{\varepsilon^{2}}{2}\|v\|^{2} .
\end{gathered}
$$

From the above, we have

$$
\frac{d}{d t}\left[\|\nu\|^{2}+\mu\left\|D^{m} u\right\|^{2}+\varepsilon^{2}\|u\|^{2}\right]+\left(\frac{\beta \lambda_{1}^{m}}{2}-2 \varepsilon-\varepsilon^{2}\right)\|\nu\|^{2}+\left(2 \varepsilon \mu_{0}-\beta \varepsilon^{2}-\frac{2 C_{0}^{2}}{\beta \lambda_{1}^{2(m-1)}}\right)\left\|D^{m} u\right\|^{2}+2 \varepsilon^{3}\|u\|^{2} \leq \frac{\|q(x) \dot{W}\|^{2}}{\varepsilon^{2}}=C_{1} .
$$

From hypothesis $(\mathrm{H} 1),(\mathrm{H} 2)$, we can get

$$
a_{1}=\frac{\beta \lambda_{1}^{m}}{2}-2 \varepsilon-\varepsilon^{2} \geq 0, \quad a_{2}=2 \varepsilon \mu_{0}-\beta \varepsilon^{2}-\frac{2 C_{0}^{2}}{\beta \lambda_{1}^{2(m-1)}} \geq 0 .
$$

Then take $\alpha_{1}=\min \left\{a_{1}, \frac{a_{2}}{\mu}, 2 \varepsilon\right\}$, then (3.17) can be transformed into

$$
\frac{d}{d t}\left(\|v\|^{2}+\mu\left\|D^{m} u\right\|^{2}+\varepsilon^{2}\|u\|^{2}\right)+\alpha_{1}\left(\|v\|^{2}+\mu\left\|D^{m} u\right\|^{2}+\varepsilon^{2}\|u\|^{2}\right) \leq C_{1} .
$$

From Gronwall's inequality

$$
\left(\|v\|^{2}+\mu\left(\left\|D^{m} u\right\|^{2}\right)+\varepsilon^{2}\|u\|^{2}\right) \leq\left(\left\|v_{0}\right\|^{2}+\mu\left(\left\|D^{m} u_{0}\right\|^{2}\right)+\varepsilon^{2}\left\|u_{0}\right\|^{2}\right) e^{-\alpha_{1} t}+\frac{C_{1}}{\alpha_{1}}\left(1-e^{-\alpha_{1} t}\right) .
$$

By hypothesis (H1), there are

$$
\|(u, v)\|_{E_{0}}^{2}=\left\|D^{m} u\right\|^{2}+\|v\|^{2} \leq\left(\left\|v_{0}\right\|^{2}+\mu\left\|D^{m} u_{0}\right\|^{2}+\varepsilon^{2}\left\|u_{0}\right\|^{2}\right) e^{-\alpha_{1} t}+\frac{C_{1}}{\alpha_{1}}\left(1-e^{-\alpha_{1} t}\right) .
$$

There exiet $C\left(R_{1}\right)$ and $t=t_{1}(\Omega)>0$, such that

$$
\|(u, v)\|_{E_{0}}^{2}=\left\|D^{m} u\right\|^{2}+\|v\|^{2} \leq C\left(R_{1}\right) . \quad\left(t>t_{1}\right) .
$$

Therefore, Lemma 1 is proved.

Lemma 2 Let $E_{k}=H_{0}^{m+k}(\Omega) \times H_{0}^{k}(\Omega),(k=1,2, \cdots m)$, for $\forall y=\left(y_{1}, y_{2}\right)^{T} \in E_{k}$, we have

$$
(L y, y)_{E_{k}} \geq k_{1}\|y\|_{E_{k}}^{2}+k_{2}\left\|D^{m+k} y_{2}\right\|^{2} .
$$

Where $k_{1}=\min \left\{\frac{\beta \varepsilon+\varepsilon-\varepsilon^{2} \lambda_{1}^{-m}}{2 \beta}, \frac{\beta \lambda_{1}^{m}-\beta \varepsilon^{2}-2 \varepsilon}{2}\right\}, k_{2}=\frac{\beta(1-\beta \varepsilon+\varepsilon)}{2}$.

$$
\text { Proof } L=\left(\begin{array}{cc}
\left(\left(M\left(\left\|A^{\frac{m}{2}} u\right\|^{2}\right)-\beta \varepsilon\right) A^{m}+\varepsilon^{2}\right) I & \left(\beta A^{m}-\varepsilon\right) I
\end{array}\right), \quad \forall y=\left(y_{1}, y_{2}\right)^{T} \in E_{k},
$$

From hypothesis (H1), Holder inequality, Young inequality and Poincare inequality

$$
\begin{aligned}
& \left.\left.(L y, y)_{E_{k}}=\left(D^{m+k}\left(\varepsilon y_{1}-y_{2}\right), D^{m+k} y_{1}\right)+\left(D^{k}\left(\left((M)\left\|A^{\frac{m}{2}} u\right\|^{2}\right)-\beta \varepsilon\right) A^{m}+\varepsilon^{2}\right) y_{1}+\left(\beta A^{m}-\varepsilon\right) y_{2}\right), D^{k} y_{2}\right) \\
& =\varepsilon\left\|D^{m+k} y_{1}\right\|-\left(D^{m+k} y_{2}, D^{m+k} y_{1}\right)+\left(M\left(\left\|A^{\frac{m}{2}} u\right\|^{2}\right) D^{m+k} y_{1}, D^{m+k} y_{2}\right)-\beta \varepsilon\left(D^{m+k} y_{1}, D^{m+k} y_{2}\right) \\
& +\varepsilon^{2}\left(D^{k} y_{1}, D^{k} y_{2}\right)+\beta\left\|D^{m+k} y_{2}\right\|-\varepsilon\left\|D^{k} y_{2}\right\|^{2}
\end{aligned}
$$




$$
\begin{aligned}
= & \varepsilon\left\|D^{m+k} y_{1}\right\|-\left(D^{m+k} y_{2}, D^{m+k} y_{1}\right)+\left(M\left(\left\|A^{\frac{m}{2}} u\right\|^{2}\right)-\varepsilon\right)\left(D^{m+k} y_{1}, D^{m+k} y_{2}\right)+(\varepsilon-\beta \varepsilon)\left(D^{m+k} y_{1}, D^{m+k} y_{2}\right) \\
& +\varepsilon^{2}\left(D^{k} y_{1}, D^{k} y_{2}\right)+\beta\left\|D^{m+k} y_{2}\right\|-\varepsilon\left\|D^{k} y_{2}\right\|^{2} \\
\geq & \varepsilon\left\|D^{m+k} y_{1}\right\|-\left(D^{m+k} y_{2}, D^{m+k} y_{1}\right)+\left(D^{m+k} y_{1}, D^{m+k} y_{2}\right)-(\beta \varepsilon-\varepsilon)\left(D^{m+k} y_{1}, D^{m+k} y_{2}\right)+\varepsilon^{2}\left(D^{k} y_{1}, D^{k} y_{2}\right) \\
& +\beta\left\|D^{m+k} y_{2}\right\|-\varepsilon\left\|D^{k} y_{2}\right\|^{2} \\
\geq & \varepsilon\left\|D^{m+k} y_{1}\right\|-\frac{(\beta-1) \varepsilon}{2 \beta}\left\|D^{m+k} y_{1}\right\|^{2}-\frac{\beta(\beta-1) \varepsilon}{2}\left\|D^{m+k} y_{2}\right\|^{2}-\frac{\varepsilon^{2}}{2 \beta}\left\|D^{k} y_{1}\right\|^{2}-\frac{\beta \varepsilon^{2}}{2}\left\|D^{k} y_{2}\right\|^{2}+\beta\left\|D^{m+k} y_{2}\right\|-\varepsilon\left\|D^{k} y_{2}\right\|^{2} \\
\geq & \frac{\beta \varepsilon+\varepsilon}{2 \beta}\left\|D^{m+k} y_{1}\right\|^{2}+\frac{\beta(1-\beta \varepsilon+\varepsilon)}{2}\left\|D^{m+k} y_{2}\right\|^{2}-\frac{\varepsilon^{2} \lambda_{1}^{-m}}{2 \beta}\left\|D^{m+k} y_{1}\right\|^{2}+\frac{\beta \lambda_{1}^{m}-\beta \varepsilon^{2}-2 \varepsilon}{2}\left\|D^{k} y_{2}\right\|^{2} \\
= & \frac{\beta \varepsilon+\varepsilon-\varepsilon^{2} \lambda_{1}^{-m}}{2 \beta}\left\|D^{m+k} y_{1}\right\|^{2}+\frac{\beta(1-\beta \varepsilon+\varepsilon)}{2}\left\|D^{m+k} y_{2}\right\|^{2}+\frac{\beta \lambda_{1}^{m}-\beta \varepsilon^{2}-2 \varepsilon}{2}\left\|D^{k} y_{2}\right\|^{2} \\
\geq & k_{1}\left(\left\|D^{m+k} y_{1}\right\|^{2}+\left\|D^{k} y_{2}\right\|^{2}\right)+k_{2}\left\|D^{m+k} y_{2}\right\|^{2} \quad=k_{1}\|y\|_{E_{k}}^{2}+k_{2}\left\|D^{m+k} y_{2}\right\|^{2} .
\end{aligned}
$$

Therefore, Lemma 2 is proved.

Lemma 3 Let $\varphi$ be a solution of the problem (3.2), then there exists a bounded random compact set $\widetilde{B}_{0 k}(w) \in D\left(E_{k}\right)$, so that for any temperedly random set $B_{k}(w) \in D\left(E_{k}\right)$, there exists a random variable $T_{B_{k}(w)}>0$, such that

$$
\varphi\left(t, \theta_{t} w\right) B_{k}\left(\theta_{-t} w\right) \subset \widetilde{B}_{0 k}(w), \quad \forall t \geq T_{B_{k}(w)}, w \in \Omega .
$$

Proof Let $\psi$ be a solution of the problem (3.3). By taking the inner product of $\psi=(u, z)^{T} \in E_{k}$ and equation (3.3) on $E_{k}$, we can get.

$$
\frac{1}{2} \frac{d}{d t}\|\psi\|_{E_{k}}^{2}+(L \psi, \psi)=\left(\bar{F}\left(\theta_{t} w, \psi\right), \psi\right) .
$$

From Lemma 2

$$
(L \psi, \psi)_{E_{k}} \geq k_{1}\|\psi\|_{E_{k}}^{2}+k_{2}\left\|D^{m+k} z\right\|^{2},
$$

$$
\begin{gathered}
\left(\bar{F}\left(\theta_{t} w, \psi\right), \psi\right)=\left(D^{m+k} q(x) \delta\left(\theta_{t} w\right), D^{m+k} u\right)+\left(D^{k}\left(-\Delta g(u)+\left(\varepsilon+1-\beta A^{m}\right) q(x) \delta\left(\theta_{t} w\right)\right), D^{k} z\right) \\
\left(D^{m+k} q(x) \delta\left(\theta_{t} w\right), D^{m+k} u\right) \leq \frac{\varepsilon}{2}\left\|D^{m+k} u\right\|^{2}+\frac{\lambda_{1}^{-m}}{2 \varepsilon}\left\|A^{m+\frac{k}{2}} q(x)\right\|^{2}\left|\delta\left(\theta_{t} w\right)\right|^{2} \\
\left.\left(D^{k} \varepsilon q(x) \delta\left(\theta_{t} w\right)\right), D^{k} z\right) \leq \frac{\varepsilon \lambda_{1}^{-m}}{2}\left\|D^{m+k} z\right\|^{2}+\frac{\varepsilon}{2}\left\|D^{k} q(x)\right\|^{2}\left|\delta\left(\theta_{t} w\right)\right|^{2} \\
\left.\left(D^{k}\left(1-\beta A^{m}\right) q(x) \delta\left(\theta_{t} w\right), D^{k} z\right) \leq \frac{\varepsilon \lambda_{1}^{-m}}{2}\left\|D^{m+k} z\right\|^{2}+\frac{1}{2 \varepsilon}\left\|D^{k} q(x)\right\|^{2}+\beta^{2}\left\|A^{m+\frac{k}{2}} q(x)\right\|^{2}\right)\left|\delta\left(\theta_{t} w\right)\right|^{2}
\end{gathered}
$$

According to hypothesis (H2), lemma 1 can be obtained

$$
\left(D^{k}(-g \Delta(u)), D^{k} z\right) \leq \frac{1}{2}\left\|g^{\prime \prime}(u)\right\|_{\infty}^{2}\|D u\|_{4}^{4}+\frac{1}{2}\left\|g^{\prime}(u)\right\|_{\infty}^{2}\|\Delta u\|^{2}+\frac{1}{2}\left\|D^{2 k} z\right\|^{2} .
$$

By interpolating inequalities, there are

$$
\begin{gathered}
\|D u\|_{L^{4}} \leq C_{2}\left\|D^{m} u\right\|_{L^{2}}^{\frac{n+4}{4 m}}\|u\|_{L^{2}}^{\frac{4 m-n-4}{4 m}} . \\
\left(D^{k}(-\Delta g(u)), D^{k} z\right) \leq C_{3}\left(\left\|g^{\prime \prime}(u)\right\|_{\infty},\left\|g^{\prime}(u)\right\|_{\infty},\left\|D^{m} u\right\|\right)+\frac{1}{2}\left\|D^{k} z\right\|^{2} \leq C_{3}+\frac{\lambda_{1}^{-(m-k)}}{2}\left\|D^{m+k} z\right\|^{2} .
\end{gathered}
$$


From the above, we have

$$
\begin{gathered}
\frac{d}{d t}\|\psi\|_{E_{k}}^{2}+2 k_{1}\|\psi\|_{E_{k}}^{2}+\left(2 k_{2}-2 \varepsilon \lambda_{1}^{-m}-\lambda_{1}^{-(m-k)}\right)\left\|D^{m+k} z\right\|^{2} \leq \varepsilon\left\|D^{m+k} u\right\|^{2}+2 C_{3}+\left.\frac{\beta^{2}+\lambda_{1}^{-m}}{\varepsilon}\left\|A^{m+\frac{k}{2}} q(x)\right\|\right|^{2}\left|\delta\left(\theta_{t} w\right)\right|^{2} \\
+\left(\varepsilon+\frac{1}{\varepsilon}\right)\left\|D^{k} q(x)\right\|^{2}\left|\delta\left(\theta_{t} w\right)\right|^{2} .
\end{gathered}
$$

where $\eta=2 k_{1}, \quad M=\frac{\beta^{2}+\lambda_{1}^{-m}}{\varepsilon}\left\|A^{m+\frac{k}{2}} q(x)\right\|^{2}+\left(\varepsilon+\frac{1}{\varepsilon}\right)\left\|D^{k} q(x)\right\|^{2}$, we have

$$
\frac{d}{d t}\|\psi\|_{E_{k}}^{2}+\eta\|\psi\|_{E_{k}}^{2} \leq C_{4}+M\left|\delta\left(\theta_{t} w\right)\right|^{2} .
$$

According to Gronwall inequation, $P_{\text {a.e.w }} \in \Omega$, we can get

$$
\|\psi(t, w)\|_{E_{k}}^{2} \leq e^{-\eta t}\left\|\psi_{0}(w)\right\|_{E_{k}}^{2}+\int_{0}^{t} e^{-\eta(t-r)}\left(C_{4}+M\left|\delta\left(\theta_{r} w\right)\right|^{2}\right) d r .
$$

Because $\delta\left(\theta_{t} w\right)$ is tempered, and $\delta\left(\theta_{t} w\right)$ is continuous with respect to $t$, according to literature Massatt, P.(1988), a temper random variable $r_{1}: \Omega \rightarrow R^{+}$can be obtained, so that for $\forall t \in R, w \in \Omega$, there is

$$
\left|\delta\left(\theta_{t} w\right)\right|^{2} \leq r_{1}\left(\theta_{t} w\right) \leq e^{\frac{\eta}{2}|t|} r_{1}(w) .
$$

Substituting $w$ by $\theta_{-t} w$ in formula (3.38),we know

$$
\left\|\psi\left(t, \theta_{-t} w\right)\right\|_{E_{k}}^{2} \leq e^{-\eta t}\left\|\psi_{0}\left(\theta_{-t} w\right)\right\|_{E_{k}}^{2}+\int_{0}^{t} e^{-\eta(t-r)}\left(C_{4}+M\left|\delta\left(\theta_{r-t} w\right)\right|^{2}\right) d r
$$

Let $r-t=\tau$

$$
\int_{0}^{t} e^{-\eta(t-r)}\left(C_{4}+M\left|\delta\left(\theta_{r-t} w\right)\right|^{2}\right) d r=\int_{-t}^{0} e^{\eta \tau}\left(C_{4}+M\left|\delta\left(\theta_{\tau} w\right)\right|^{2}\right) d \tau \leq \frac{C_{4}}{\eta}+\frac{2}{\eta} M r_{1}(w)
$$

Because $\varphi_{0}\left(\theta_{-t} w\right) \in B_{k}\left(\theta_{-t} w\right)$ is tempered, and $\left|\delta\left(\theta_{-t} w\right)\right|$ is also tempered, hence we let

$$
R_{0}^{2}(w)=\frac{C_{4}}{\eta}+\frac{2}{\eta} M r_{1}(w)
$$

Then $R_{0}^{2}(w)$ is also tempered, $\hat{B}_{0 k}=\left\{\psi \in E_{k}\|\psi \psi\|_{E_{k}} \leq R_{0}(w)\right\}$ is called a random absorption set, because

$$
\tilde{S}\left(t, \theta_{-t} w\right) \psi_{0}\left(\theta_{-t} w\right)=\varphi\left(t, \theta_{-t} w\right)\left(\psi_{0}\left(\theta_{-t} w\right)+\left(0, q(x) \delta\left(\theta_{-t} w\right)\right)^{T}\right)-\left(0, q(x) \delta\left(\theta_{-t} w\right)\right)^{T}
$$

so let

$$
\tilde{B}_{0 k}(w)=\left\{\varphi \in E_{k}\|\varphi\|_{E_{k}} \leq R_{0}(w)+\left\|D^{k} q(x) \delta(w)\right\|=\bar{R}_{0}(w)\right\}
$$

Then $\tilde{B}_{0 k}(w)$ is the random absorption set of $\varphi(t, w)$, and $\widetilde{B}_{0 k}(w) \in D\left(E_{k}\right)$. The proof is complete.

Lemma 4 When $k=m$, for $\forall B_{m}(w) \in D\left(E_{m}\right)$, let $\varphi(t)$ be the solution of equation (3.2) in initial value $\varphi_{0}=\left(u_{0}, u_{1}+\varepsilon u_{0}\right)^{T} \in B_{m}$. It can be decomposed into $\varphi=\varphi_{1}+\varphi_{2}$, where $\varphi_{1}, \varphi_{2}$ satisfy respectively

$$
\begin{gathered}
\left\{\begin{array}{l}
d \varphi_{1}+L \varphi_{1} d t=0, \\
\varphi_{01}(w)=\left(u_{0}, u_{1}+\varepsilon u_{0}\right)^{T}
\end{array}\right. \\
\left\{\begin{array}{l}
d \varphi_{2}+L \varphi_{2} d t=F(w, \varphi), \\
\varphi_{02}(w)=0
\end{array}\right.
\end{gathered}
$$


Then

$$
\left\|\varphi_{1}\left(t, \theta_{-t} w\right)\right\|_{E_{m}}^{2} \rightarrow 0,(t \rightarrow \infty), \quad \forall \varphi_{0}\left(\theta_{-t} w\right) \in B_{m}\left(\theta_{-t} w\right),
$$

and there exists a temper random radius $R_{1}(w)$, which satisfies for $w \in \Omega$.

$$
\left\|\varphi_{2}\left(t, \theta_{-t} w\right)\right\|_{E_{m}} \leq R_{1}(w)
$$

Proof Let $\psi=\psi_{1}+\psi_{2}=\left(u_{1}, u_{1 t}+\varepsilon u_{1}\right)^{T}+\left(u_{2}, u_{2 t}+\varepsilon u_{2}-q(x) \delta\left(\theta_{t} w\right)\right)^{T}$ be the solution of equation (3.3), then according to equation (3.45) and (3.46), we know that $\psi_{1}, \psi_{2}$ satisfy respectively

$$
\begin{aligned}
& \left\{\begin{array}{c}
\psi_{1 t}+L \psi_{1}=0 \\
\psi_{01}=\psi_{0}=\left(u_{0}, u_{1}+\varepsilon u_{0}-q(x) \delta\left(\theta_{t} w\right)\right)^{T}
\end{array}\right. \\
& \left\{\begin{array}{c}
\psi_{2 t}+L \psi_{2}=\bar{F}\left(\psi_{2}, \theta_{t} w\right), \\
\psi_{02}=0
\end{array}\right.
\end{aligned}
$$

By taking the inner product of equation $\psi_{1}=\left(u_{1}, u_{1 t}+\varepsilon u_{1}\right)^{T}$ and equation (3.49) on $E_{m}$ we can get

$$
\frac{1}{2} \frac{d}{d t}\left\|\psi_{1}\right\|_{E_{m}}^{2}+\left(L \psi_{1}, \psi_{1}\right)=0
$$

According to lemma 2 and Gronwall inequality, we have

$$
\left\|\psi_{1}(t, w)\right\|_{E_{m}}^{2} \leq e^{-2 k_{1} t}\left\|\psi_{01}(w)\right\|_{E_{m}}^{2} .
$$

Substituting $w$ by $\theta_{-t} w$ in formula (3.52), and because $\delta\left(\theta_{-t} w\right) \in B_{m}$ is tempered, then

$$
\left\|\psi_{1}\left(t, \theta_{-t} w\right)\right\|_{E_{m}}^{2} \leq e^{-2 k_{1} t}\left\|\psi_{0}\left(\theta_{-t} w\right)\right\|_{E_{m}}^{2} \rightarrow 0,(t \rightarrow \infty), \quad \forall \psi_{0}\left(\theta_{-t} w\right) \in B_{m} .
$$

Thus (3.47) is hold. The inner product of $\psi_{2}=\left(u_{2}, u_{2 t}+\varepsilon u_{2}-q(x) \delta\left(\theta_{t} w\right)\right)^{T}$ and equation (3.50) on $E_{m}$ is obtained according to Lemma 1, Lemma 2 and Lemma 3

$$
\frac{d}{d t}\left\|\psi_{2}\right\|_{E_{m}}^{2}+\eta\left\|\psi_{2}\right\|_{E_{m}}^{2} \leq C_{4}+M_{1}\left|\delta\left(\theta_{t} w\right)\right|^{2} .
$$

Where $\eta=2 k_{1}, \quad M_{1}=\frac{\beta^{2}+\lambda_{1}^{-m}}{\varepsilon}\left\|A^{\frac{3 m}{2}} q(x)\right\|^{2}+\left(\varepsilon+\frac{1}{\varepsilon}\right)\left\|D^{m} q(x)\right\|^{2}$,

Substituting $\theta_{-t} w$ for $w$ in Formula (3.54) is obtained by Gronwall inequality, we have

$$
\left\|\psi_{2}\left(t, \theta_{-t} w\right)\right\|_{E_{m}}^{2} \leq e^{-\eta t}\left\|\psi_{02}\left(\theta_{-t} w\right)\right\|_{E_{m}}^{2}+\int_{0}^{t} e^{-\eta(t-r)}\left(C_{4}+M_{1}\left|\delta\left(\theta_{r-t} w\right)\right|^{2}\right) d r \leq \frac{C_{4}}{\eta}+\frac{2}{\eta} M_{1} r_{1}(w) .
$$

So there is a tempered random radius

$$
R_{1}^{2}(w)=\frac{C_{4}}{\eta}+\frac{2}{\eta} M_{1} r_{1}(w)
$$

So that for $\forall w \in \Omega$, there is

$$
\left\|\varphi_{2}\left(t, \theta_{-t} w\right)\right\|_{E_{m}} \leq R_{1}(w)
$$

Therefore, Lemma 4 is proved.

Lemma 5 The identified stochastic dynamic system $\{S(t, w), t \geq 0\}$ determined by equation (3.2) has a compact absorption set $K(w) \subset E_{k} \mathrm{C}$ in $t=0, P_{\text {a.e. }} \in \Omega$.

Proof Let $K(w)$ be a closed sphere with radius $R_{1}(w)$ in space $E_{k}$. According to embedding relation $E_{k} \subset E_{0}, K(w)$ is a compact set in $E_{k}$. For arbitrary temper random set $B_{k}(w)$ in $E_{k}$, for $\forall \varphi\left(t, \theta_{-t} w\right) \in B_{k}$, according to lemma 4, 


$$
\begin{aligned}
& \varphi_{2}=\varphi-\varphi_{1} \in K(w), \text { so for } \forall t \geq T_{B_{k}(w)}>0 \text {, we have } \\
& \qquad \begin{array}{c}
d_{E_{k}}\left(S\left(t, \theta_{-t} w\right) B_{k}\left(\theta_{-t} w\right), K(w)\right)=\inf _{\vartheta(t) \in K(w)}\left\|\varphi\left(t, \theta_{-t} w\right)-\vartheta(t)\right\|_{E_{k}}^{2} \\
\leq\left\|\varphi_{1}\left(t, \theta_{-t} w\right)\right\|_{E_{k}}^{2} \\
\leq e^{-2 \eta t}\left\|\varphi_{01}\left(\theta_{-t} w\right)\right\|_{E_{k}}^{2} \rightarrow 0,(t \rightarrow \infty) .
\end{array}
\end{aligned}
$$

Therefore, Lemma 5 is proved.

According to Lemma 1-Lemma 5, there are the following theorems.

Theorem 1 The random dynamical $\operatorname{system}\{S(t, w), t \geq 0\}$ has a family of random attractors $A_{k}(w) \subset K(w) \subset E_{k}$, $w \in \Omega$ and there exists a tempered random set $K(w)$, so that $P_{\text {a.e.w }} \in \Omega$

$$
A_{k}(w)=\bigcap_{t \geq 0, \tau \geq t} \overline{U S\left(t, \theta_{-\tau} w, K\left(\theta_{-\tau} w\right)\right)} .
$$

And $S(t, w) A_{k}(w)=A_{k}\left(\theta_{t} w\right)$. The proof is complete.

\section{Acknowledgements}

We express our sincere thanks to the anonymous reviewer for his/her careful reading of the paper, we hope that we can get valuable comments and suggestions. Making the paper better.

\section{References}

Cai, D. H., Fan, X. M., \& Ye, J. J. (2014). Considering the stochastic attractor of dissipative KdV equation with multiplicative noise. Journal of Southwest Nationalities University, (Natural Science Edition), 40(6), 900-904. https://doi.org/10.3969/j.issn.1003-4271.2014.06.18

Cheng, Y. Y., \& Li, Y. R. (2012). Random attractants for the generalized kuranoto-sivashinsky equation with white noise. Journal of Southwest Normal University, 37(10), 26-30. https://doi.org/10.13718/j.cnki. Xsxb.2012.10.027

Guo, B. L. (2000). Infinite dimensional dynamic system. National Defense Industry Press.

Guo, B. L., \& Pu, X. K. (2009). Random infinite dimensional dynamics dystem . Beijing University of Aeronautics and Astronautics Press.

Guo, B. L., \& Wang, B. X. (1995) .Finite dimensional behavior for the derivative ginzburg-landau equation in two spatial dimensions. Journal of Physics D, 89, 83-90. https://doi.org/10.1016/0167-2789(95)00216-2

Hao, H. J., \& Zhou, S. F. (2010). Existence of stochastic attractors for stochastic sine-Gordon equation with strong damping. Journal of Shanghai Normal University (Natural Science Edition), 39(2), 121-127.

Lin, G. G. (2011). Nonlinear evolution equation. Yunnan University Press.

Lin, G. G., Chen, L., \& Wang, W. (2017). Random attractors of the stochastic strongly damped for the higher-order nonlinear Kirchhoff-type equation. International Journal of Modern Nonlinear Theory and Application, 6, 59-69. https://doi.org/10.4236/ijmnta.2017.62005

Massatt, P. (1988). Limiting behaviour for a strong damped nonlinear wave equation. Journal of Differential Equations, (48), 334-349. https://doi.org/10.1016/0022-0396(83)90098-0

Qin, C. L., \& Du, J. J. (2016). Stochastic attractor of stochastic Kirchhoff equation with strong damping. Journal of Science of Normal University, 36(12), 6-11. https://doi.org/10.3969/j.issn.1007-9831.2016.12.002

Qin, C. L., Du, J. J., \& Lin, G. G. (2017). Random attractors for the Kirchhoff-type suspension bridge equation with strong damping and white noises. International Journal of Modern Nonlinear Theory and Application, 6, 134-147. https://doi.org/10.4236/ijmnta.2017.64012

Wang, M. (2017). Stochastic attractor of strongly damped stochastic wave equation. Journal of Zhejiang Normal University, 5, 17-27.

Wang, R., \& Li, Y. R. (2012). Random attractor of generalized Ginzbury-Landau equation with multiplicative white noise. Journal of Southwest University (Natural Science Edition), 34(2), 92-95. https://doi.org/10.13718/j.cnki. xdzk.2012.02.07

Xu, G. G., Wang, L. B., \& Lin, G. G. (2017). Random attractors for nonautonomous stochastic wave equations with 
dispersion and dissipation terms. Journal of Applied Functional Analysis, 19(3), 231-249. https://doi.org/10.12012/1009-1327(2017)03-0231-19

Yin, J. Y., Li, Y. R., \& Zhao, H. J. (2013). Dissipative hamilitonian amplitude modulation wave instability with white noise. Journal of Southwest Normal University (Natural Science Edition), 38(4), 44-48. https://doi.org/10.13718/j.cnki. xsxb.2013.04.036

Zhao, C. D., \& Zhou, S. F. (2009). Sufficient conditions for the existence of global random attractors for stochatic lattice dunamical systems and applications. Journal of Mathematical Analysis and Applications, 54, 78-95. https://doi.org/10.1016/j.jmaa.2008.12.036

\section{Copyrights}

Copyright for this article is retained by the author(s), with first publication rights granted to the journal.

This is an open-access article distributed under the terms and conditions of the Creative Commons Attribution license (http://creativecommons.org/licenses/by/4.0/). 